\title{
Enseñanza y aprendizaje del concepto de corriente eléctrica basado en analogías y metáforas
}

\section{Teaching and learning of the electrical current concept based on analogies and metaphors}

\section{Lehr- und lernkonzept von strom durch analogien und metaphern}

Hernando González ${ }^{1}$, Gonzalo Marín², Fernanda Ortiz ${ }^{3}$

\section{Resumen}

La elaboración y aplicación de estrategias metodológicas, basadas en el uso de analogías y metáforas, sobre el tema de corriente eléctrica en el curso de Física de los estudiantes de grado 11 pertenecientes al Instituto Técnico Superior de la ciudad de Neiva, produjo buenos resultados de acuerdo con las pruebas efectuadas para evaluar el aprendizaje significativo de los estudiantes, mediante mapas conceptuales y redes semánticas.

Palabras clave : Aprendizaje significativo, analogías, mapa conceptual, corriente eléctrica

\section{Zusammenfassung}

Wir Gebrauchten Analogien und Metaphern, wie Methodische Strategien zur Accomplished ein Verständnis des elektrischen Stroms Konzept im Physikkurs für Schüler der 11. Klasse aus der Höheren Technischen Institut der Neiva Stadt.

\section{Abstract}

We used analogies and metaphors, as methodological strategies to accomplish an understanding of the electrical current concept en the Physics course for students of 11th grade from the Superior Technical Institute of Neiva city.

Keywords: Meaningful learning, Analogies, Concept map, Electrical current
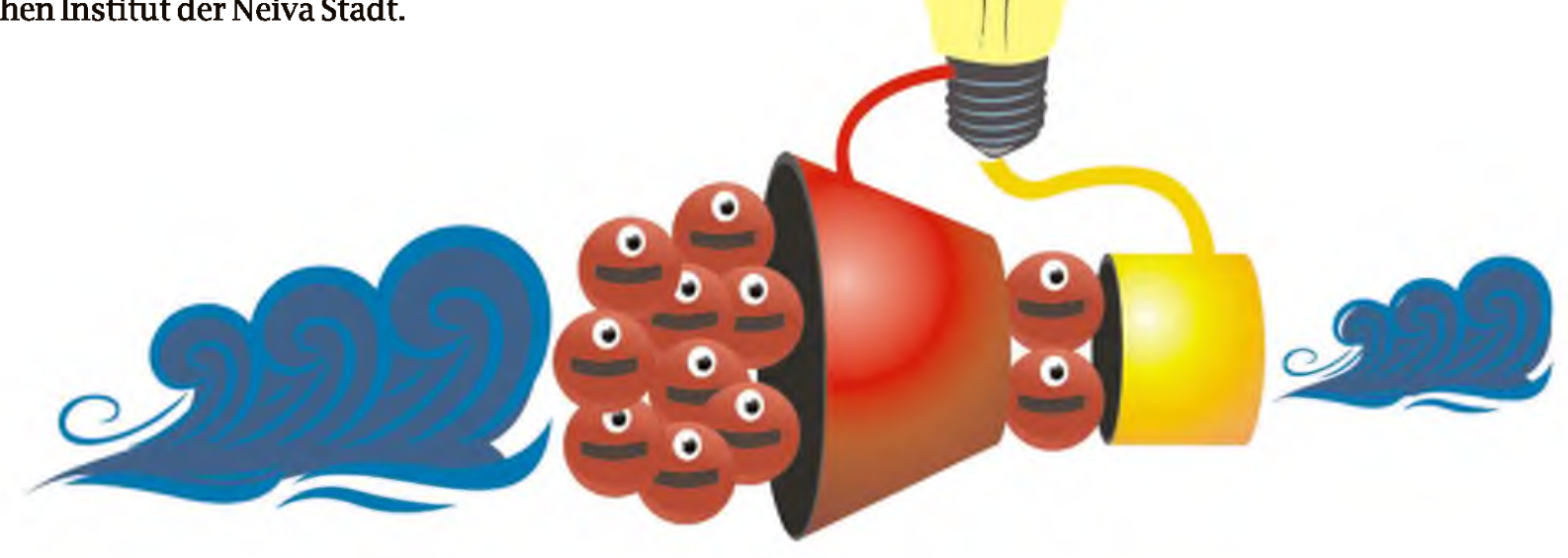

1 - Doctor en Física. Docente Facultad de Ciencias Exactas y Naturales. Universidad Surcolombiana. Neiva. Colombia. hergosi@usco.edu.co 2 - Licenciado en Educación Básica con énfasis en Ciencias Naturales y Educación Ambiental. Universidad Surcolombiana. Neiva. Colombia. gonzalomarinoviedo123@gmail.com

3 - Licenciada en Educación Básica con énfasis en Ciencias Naturales y Educación Ambiental. Universidad Surcolombiana. Neiva. Colombia. fer2590@hotmail.com 


\section{Introducción}

El uso de analogías, analogías estructurales, metáforas y símiles es una potente herramienta para diseñar estrategias metodológicas en la enseñanza de las Ciencias, y en particular, en la enseñanza de la Física (Linares e Izquierdo, 2006; Russel, 2009).

No es fácil emplear continuamente estas elaboraciones para ser utilizadas frecuentemente dentro del aula de clase, debido a diferentes factores (Furió y Guisasola, 1999; Zamorano et al, 2006; González y Valcárcel, 2009). En este artículo presentamos un trabajo de elaborado por estudiantes de Licenciatura en Educación Básica con énfasis en Ciencias Naturales y Educación Ambiental, usando en un diseño metodológico basado en analogías y metáforas, para procurar el aprendizaje del concepto de corriente eléctrica, en estudiantes de grado 11 del Instituto Técnico Superior de la ciudad de Neiva.

La organización de este artículo es la siguiente: En la sección 2 presentamos las diferencias y semejanzas entre analogías y metáforas, fundamentadas principalmente en el entendimiento que de ellas se tiene en el contexto de la enseñanza de las Ciencias (Moreira y Greca, 2003; Pérez, 2013). En la sección 3 ilustramos la organización de las estrategias metodológicas elaboradas para llevar a cabo el estudio. En la sección 4 se dan a conocer los resultados sobre el uso de analogías y metáforas empleadas en el aula de clase, para lograr el aprendizaje significativo del concepto de corriente eléctrica (Novak, 1988). La sección 5 contiene un análisis sobre los resultados obtenidos y en la última sección se dan las conclusiones, las recomendaciones y aspectos relacionados.

\section{Analogías y Metáforas}

En términos genéricos el concepto de analogía consiste en buscar correspondencias de comparación entre los distintos elementos de dos campos del conocimiento, según lo expresan Linares e Izquierdo (2006). En la elaboración de nuevas teorías y modelos es frecuente la utilización de analogías provenientes de diferentes campos del conocimiento; entre otros aspectos, el uso de analogías produce el fortalecimiento del pensamiento creativo y es de gran utilidad en el campo de enseñanza de las ciencias.

La analogía estructural es un concepto extraído de la Mecánica Clásica basado en la universalidad de los principios variacionales (Goldstein, 2002). En alusión al párrafo anterior, es conocido que las ecuaciones de movimiento para un sistema mecánico, por ejemplo, se pueden obtener a partir de las Leyes de Newton o a partir de las ecuaciones de Lagrange o Hamilton. Desde otro punto de vista, es frecuente encontrar que algunos sistemas, pertenecientes a diversos marcos de la Física, digamos un sistema mecánico masaresorte en un medio viscoso y un circuito eléctrico inductancia-resistencia, obedecen ecuaciones diferen- ciales similares de movimiento, por lo cual es usual decir que ellos presentan una analogía.

Las metáforas son construcciones del lenguaje para mostrar algún rasgo de utilidad entre diferentes conceptos. Se considera la metáfora como una deformación del lenguaje, pero muchas veces se utiliza para buscar complementos a conceptos que son abstractos o poco entendibles, usando el lenguaje propio del contexto donde se ubican (Aguilar y Arroyo, 2008). Una muestra es plausible cuando tratamos de dar sentido real al concepto de espín en la mecánica cuántica y lo asociamos al movimiento de rotación de la tierra sobre su propio eje; sin embargo, se debe entender que el concepto de espín tiene un origen completamente distinto al de movimiento de rotación alrededor de un punto fijo y que no podemos mezclar estos dos aspectos.

Aunque presentamos un análisis y características básicas de analogías y metáforas, la investigación se basó en el uso de ellas como herramientas de Enseñanza y Aprendizaje (E-A) en el aula de clase. Es de resaltar que estas formas de EA no son usadas frecuentemente por los docentes; en términos generales, las investigaciones sobre esta temática, en particular, son muy escasas en nuestro país

\section{Metodología}

Las dificultades sobre el aprendizaje del concepto de corriente eléctrica en la escuela secundaria, la indagación acerca de cómo contribuir a su mejoramiento y la elección de analogías y metáforas como estrategias metodológicas de EA, nos condujeron al planteamiento del siguiente interrogante en nuestro trabajo de grado: ¿Cómo diseñar y aplicar una guía didáctica para la enseñanza del concepto de corriente eléctrica basada en el uso de analogías y metáforas para los estudiantes del grado 1103 jornada de la mañana de la Institución Educativa Técnico Superior de Neiva?

Elegimos como metodología, para dar solución a la pregunta de investigación planteada, un estudio de caso con enfoque cualitativo, dada la interpretación que se da a este proceso de EA en los estudiantes de 1103 , no puede ser captado por la Estadística o las Matemáticas, centrando el análisis en la descripción de los eventos observados.

Este proceso se desarrolló mediante tres fases correspondientes a identificación, diseño y cierre, descritas a continuación.

\section{Identificación}

En la primera fase de indagación, se llevó a cabo una observación descriptiva en el espacio de conceptualización de la corriente eléctrica, sin realizar la presentación, ni descripción de la propuesta investigativa ante los estudiantes; considerando que ello podría alterar el aprendizaje, las relaciones maestro-estudiante y estudiante-estudiante, modificando sustancialmente sus percepciones. 
Esta etapa se inicia con la aplicación de un test (Russell, 2009), (Zamorano, Gibbs y Viau, 2006) sobre los conceptos más relevantes de corriente eléctrica. El análisis de datos sobre la prueba de entrada nos permitió conocer las concepciones sobre los temas elegidos, identificando las dificultades que los estudiantes tienen en torno a aspectos relacionados con corriente eléctrica. Este test diagnostico nos permitió elegir las estrategias didácticas, analogías y metáforas, para lograr minimizar dificultades conceptuales relacionadas con el tema de estudio.

Los aspectos explorados están relacionados con: Trabajo hecho por una fuerza, energía cinética, energía potencial gravitatoria, energía, corriente eléctrica, resistencia eléctrica y potencial eléctrico (Tippens, 1999), (Young y Freedman, 2009).

\section{Diseño}

En la segunda fase de la investigación se diseñaron y aplicaron las herramientas de intervención (analogías y metáforas) para lograr un mejor entendimiento de la corriente eléctrica y temas relacionados, tomando como muestra los estudiantes del grado 1103 que constituirían el caso. El proceso, en todas sus etapas o fases, fue realizado en el Instituto Técnico Superior de la ciudad de Neiva.

Usando el método de enseñanza por exposición se elaboraron y aplicaron metáforas a analogías, en los temas relevantes, durante el desarrollo de las jornadas de clases magistrales. Se estructuraron las herramientas de aprendizaje asociándolas a las definiciones de corriente eléctrica y temas complementarios. La aplicación de las herramientas quedó incluida en una guía didáctica que fue diseñada con este propósito (Marín y Ortiz, 2014).

El contenido de la guía, incluyendo las definiciones de corriente eléctrica basadas en metáforas y analogías, anexa lecturas complementarias y ejemplos específicos sobre los temas seleccionados. Como apoyo y complemento a esta labor docente, y desde la perspectiva de la incentivación a los estudiantes, se realizaron experimentos demostrativos sobre el tema complementado con una sesión de preguntas y respuestas.

En la primera parte de esta fase se presentó el esquema general de las lecciones incluyendo los temas, objetivos, el material de apoyo a la labor docente, la guía didáctica, las lecturas complementarias.

En la parte final de la primera sesión se ilustró a los estudiantes sobre la organización cognoscitiva utilizando los principios de integración de los conceptos erróneos, con los nuevos aceptados por la ciencia, promoviendo un aprendizaje de recepción activa promoviendo un enfoque crítico entre ellos a través de la elaboración de sus propios mapas conceptuales.

En la disertación expositiva de cada una de las definiciones, introducidas en forma jerárquica, en términos de analogías y metáforas, se efectuaron evaluaciones para detectar falencias en la interpretación y comprensión de los conceptos por parte de los estudiantes.

\section{Cierre}

En esta etapa de la investigación, se efectuó una interpretación de los resultados arrojados por el test de entrada, realizando una prueba en forma de mapa conceptual o red semántica teniendo en cuenta el modelo del aprendizaje significativo de Ausubel.

Desde la perspectiva de Ausebel (1976), es conocido que cuando se ha adquirido realmente un aprendizaje significativo se tiene la capacidad de efectuar una asociación conceptual en forma jerárquica. Lo anterior significa que el estudiante adquiere la habilidad de elaborar una diferenciación de naturaleza progresiva, partiendo de conceptos generales y dirigiéndose a los específicos; en otros términos, logra construir mapas conceptuales o redes semánticas, comparando los conocimientos recientemente adquiridos con los aceptados por la ciencia y va reemplazando los de su conocimiento previo, posiblemente errados, por los correctos.

Una vez aplicado este test se cualificó el caso reflexionando sobre el análisis de las respuestas y discusiones sostenidas por los estudiantes en los mapas conceptuales o redes semánticas, ello nos ilustra la dinámica mediante la cual los estudiantes modifican sus modelos sobre la corriente eléctrica que tenían anteriormente. Se elaboraron las conclusiones y recomendaciones referentes al caso estudiado y las posibles implicaciones, para el desarrollo de futuras investigaciones referidas a la enseñanza del concepto de corriente eléctrica.

\section{Resultados del Test Sobre Ideas Previas}

Antes de empezar con el desarrollo de este trabajo del aprendizaje significativo del concepto de corriente eléctrica, mediante el diseño y aplicación de analogías y metáforas, se llevó a cabo una fase de identificación descriptiva en la conceptualización, según lo planeado en la metodología, optando por indagar las ideas previas que tienen los estudiantes sobre el tema mediante un test.

Esta indagación nos aproxima a los modelos mentales que traen los estudiantes al aula, ya que manifiestan sus representaciones de lo que entienden sobre corriente eléctrica. Se pretende con este trabajo generar nuevos modelos mentales significativos de la corriente eléctrica, mediante el uso de analogías y metáforas.

El test se realizó en el aula de clase tal como, aplicándose a una muestra de 15 estudiantes en un lapso de tiempo de 20 minutos, de un total de 30 alumnos que conforman el curso 1103 de la jornada de la mañana. Los resultados fueron los siguientes:

Las preguntas en busca de la las ideas previas que tienen los estudiantes, acerca de conceptos como 
trabajo, energía y estructura atómica, pretendían detectar cómo asocian las expresiones matemáticas (Leyes de la Naturaleza) con el fenómeno físico, y cómo relacionan este último con la vida cotidiana; adicionalmente, cómo asocian las interacciones a partir de la estructura atómica de la materia. Estos aspectos básicos conformaron las preguntas incluidas en el test, que nos proporcionan su el estado de conocimiento y que permiten orientar los procedimientos de enseñanza - aprendizaje más eficientes y significativos.

Del análisis de las respuestas mencionadas, que fueron consignadas por los estudiantes, según sus ideas previas, se pudo identificar que ellos muy poco relacionan una ecuación con un aspecto físico, esto fue importante en el diseño y aplicación de las analogías y metáforas, especialmente cuando se asocian con una ecuación.

Lo expuesto en el aula de clase, sobre corriente eléctrica, se focalizó en la cotidianidad diaria de los estudiantes, tomando como referente el poco uso que ellos hacen de estas experiencias. El entendimiento de la estructura atómica de la materia es fundamental para comprender las conceptualizaciones de corriente eléctrica.

En el test también se realizaron preguntas relacionadas sobre los procesos que se llevan a cabo en el transporte de la corriente eléctrica a distintos lugares de la ciudad. Otros aspectos, incluidos en el test, versaron sobre qué entendían ellos sobre la corriente eléctrica y de que dependía para que hubiese un buen flujo eléctrico; sí consideraban que los diferentes materiales oponen diferentes resistencias o para qué sirve una batería y qué creen que ha pasado cuando una batería cesa de funcionar. Por último, se indagó sobre qué aspectos adicionales les gustaría saber sobre la corriente eléctrica. Estas preguntas nos indicaron el estado del conocimiento sobre el tema en los estudiantes facilitando las formas y recursos que les permitieran inferir aspectos relacionados con corriente eléctrica.

De las respuestas consignadas por los estudiantes se identificaron varias dificultades de aprendizaje, ya que ellos usaron definiciones erróneas para dar solución a las preguntas y usando terminología de la vida cotidiana para explicar diferentes fenómenos eléctricos que se indagaron.

Todos los aspectos analizados indicaron la necesidad de replantear la forma en que se debe de proceder para aplicar las estrategias de E-A, Por ejemplo usando análogos experimentales para reforzar esas ideas previas, y el laboratorio de Física es el lugar más propicio para hacer uso de experimentos demostrativos que incorporen analogías. Utilizando analogías que han trabajado otros autores y que han sido pertinentes en los procesos de E.A; Diseñar nuestras propias analogías para trabajar frente a las específicas dificultades de aprendizaje.
Diseño y Aplicación de la Guía Didáctica: Estrategias Metodológicas (Analogías y Metáforas) y Evaluación Una vez terminada la fase de identificación, se continuó con la fase de diseño, según lo previsto en la metodología, teniendo en cuenta el problema de investigación que se generó a partir de la pregunta problema ¿Cómo diseñar y aplicar una guía didáctica para la enseñanza del concepto de corriente eléctrica basada en el uso de analogías y metáforas para los estudiantes del grado 1103 jornada mañana de la Institución Educativa Técnico Superior? Se elaboró y aplicó la guía didáctica que anexa diversas variables como las ideas previas, el método de enseñanza expositiva, y las estrategias didácticas, de tal manera que fuese eficiente para el aprendizaje del estudiante.

La primera variable que se introdujo, en la guía didáctica, fueron las ideas previas de los estudiantes, incluyendo el tratamiento de las dificultades de aprendizaje. También se excluyeron las deducciones de las leyes y principios relevantes, para proporcionar asociaciones con conceptos y definiciones, y se usaron las ecuaciones más importantes.

La segunda variable para la elaboración de la guía está basada en el método de enseñanza expositiva: Inicialmente se presentan los objetivos de la lección, aislando las propiedades definitorias, proporcionando ejemplos, aportando un contexto y reiterando, se socializa el conocimiento y experiencia del sujeto.

Como segunda actividad se determina el material de trabajo, explicando la organización, ordenándose secuencialmente el aprendizaje manteniendo la atención de los estudiantes, y como última actividad se determina la organización cognitiva utilizando los principios de integración, promoviendo un aprendizaje de recepción activa y suscitando un enfoque critico a partir de la participación y las evaluaciones que se realicen.

El diseño de la guía didáctica está distribuido en tres momentos. Un momento de inicio que abarca todo el contenido de manera general, seguidamente el momento relacionando los conceptos con las analogías y metáforas; y un cierre donde se toma en cuenta la integración de los temas, las conclusiones y evaluaciones de los contendidos de corriente eléctrica.

Además la guía didáctica está estructurada por el propósito, que es en esencia el objetivo, por las actividades, que hacen referencia al quehacer organizado y sistematizado en una secuenciación diseñada cuidadosamente; por último, por la evaluación, donde es posible conocer el grado de aprendizaje de cada concepto. Desde esta perspectiva, el estudiante usa un enfoque crítico de las ideas previas, con las nuevas, que se generan en cada secuencia para dar una interpretación coherente a cada concepto. La guía se fundamenta bajo el objetivo general de la lección del concepto de corriente eléctrica y los objetivos específicos debido a que la enseñanza expositiva 
requiere de estos cuando se ilustra de manera general un tema.

La última variable incluida, fueron las estrategias metodológicas (analogías y metáforas) que se diseñaron, basándonos en las dificultades de los estudiantes, como por ejemplo relacionar la corriente eléctrica con las experiencias vivenciales (análogo); relacionar las ecuaciones (corriente eléctrica) con expresiones matemáticas ya conocidas (análogo), que permitan a asociarlas al fenómeno. Por último se incorporan en los análogos el trabajo de laboratorio, ya que el método de enseñanza expositivo no lo incluye en el momento de presentar los materiales de trabajo.

Una vez se aplicó esta guía didáctica en los estudiantes, se prosiguió a evaluar en forma de mapa conceptual o red semántica (Novak, 1998) sobre todo lo que habían comprendido del concepto de corriente eléctrica. De esta manera se pudo identificar el aprendizaje significativo adquirido por los estudiantes de 1103.

A continuación ilustramos, mediante un diagrama, los resultados del proceso completo de aprendizaje significativo efectuado con los estudiantes.

Diagrama 1: Cuadro comparativo entre las ideas previas de los estudiantes, proporcionadas por el test de entrada, y el test al final del estudio después de aplicar las estrategias didácticas basadas en analogías y metáforas.

\begin{tabular}{|c|c|c|}
\hline & ANTES (PRIMER TEST) & DESPUÉS (ÚLTIMO TEST) \\
\hline ENERGIA & $\begin{array}{l}\text { " Magnitud que se transforma } \\
\text { "Es la cantidad física que genera } \\
\text { movimiento } \\
\text { " Hace funcionar electrodomésticos } \\
\text { "Es una carga de energía }\end{array}$ & $\begin{array}{l}\text { * Es generada por el movimiento de } \\
\text { partículas con carga eléctrica. } \\
\text { "Es la cantidad que transporta la } \\
\text { corriente eléctrica. }\end{array}$ \\
\hline $\begin{array}{l}\text { ENERGIA } \\
\text { POTENCIAL } \\
\text { (EP) } \\
\text { ENERGIA } \\
\text { POTENCIAL } \\
\text { ELÉCTRICA } \\
\text { (U) }\end{array}$ & $\begin{array}{l}\text { " Es la energia con que se mueve } \\
\text { un objeto } \\
\text { * Es lo que se relaciona } \\
\text { con la gravedad }\end{array}$ & $\begin{array}{l}\text { * Cuando sobre una carga eléctrica se } \\
\text { realiza trabajo en contra del campo } \\
\text { eléctrico, entonces esa carga eléctrica } \\
\text { asimila energía potencial, así como un } \\
\text { cuerpo adquiere energía potencial } \\
\text { cuando se le traslada en un campo } \\
\text { gravitacional. } \\
\text { * La carga eléctrica es quien transporta } \\
\text { la energía potencial que se le } \\
\text { proporciona al electrodoméstico } \\
\text { que entrega al electrodoméstico. }\end{array}$ \\
\hline Áтомо & $\begin{array}{l}\text { " Las imágenes fueron erróneas } \\
\text { " No hubo descripción de su } \\
\text { funcionamiento. }\end{array}$ & $\begin{array}{l}\text { "El átomo contiene electrones que se } \\
\text { convierten en portadores de la } \\
\text { corriente eléctrica. } \\
\text { "La materia está compuesta de átomos } \\
\text { que alojan cargas de signos positivos } \\
\text { (protones) y negativos (electrones) }\end{array}$ \\
\hline $\begin{array}{l}\text { RESISTENCIA } \\
\text { ELEECTRICA }\end{array}$ & * Depende del material & $\begin{array}{l}\text { * Depende del material, de sus formas, } \\
\text { de la longitud y del área. } \\
\text { "Es un factor que se opone a la } \\
\text { corriente eléctrica, de la misma forma } \\
\text { que los cuerpos se oponen a que sean } \\
\text { acelerados. }\end{array}$ \\
\hline VOLTAJE & $\begin{array}{l}\text { " Hay energía + y - que forma corriente } \\
\text { " Le da potencia a la electricidad }\end{array}$ & $\begin{array}{l}\text { * Es un elemento importante para } \\
\text { producir una corriente eléctrica. Se } \\
\text { mide en voltios y es proporcionado } \\
\text { por las pilas y baterías. } \\
\text { "Es el trabajo por unidad de carga que } \\
\text { realizan fuerzas eléctricas y mueven } \\
\text { cargas del potencial mayor al menor }\end{array}$ \\
\hline $\begin{array}{l}\text { CORRIENTE } \\
\text { ELÉCTRICA }\end{array}$ & $\begin{array}{l}\text { *Es la energía utilizada para que } \\
\text { ciertos electrodomésticos funcionen } \\
\text { Es una chispa de luz y depende de la } \\
\text { fuerza y movimiento, incluso de los } \\
\text { electrones y de unos buenos cables } \\
\text { para que tenga buen flujo }\end{array}$ & $\begin{array}{l}\text { *Es un flujo de cargas eléctricas que } \\
\text { pasan por un conductor en } \\
\text { determinado tiempo } \\
\text { " Puede ser generada por circuitos de } \\
\text { tipo hidráulico, eólico, nuclear, solar. } \\
\text { "Puede ser alterna y continua } \\
\text { "Se necesita de un conductor que } \\
\text { permita el flujo en un determinado } \\
\text { tiempo. }\end{array}$ \\
\hline
\end{tabular}




\section{Conclusiones}

El diseño y aplicación de estrategias didácticas, basadas en analogías y metáforas, mediante la elaboración de una guía didáctica, facilitó la enseñanza del tema de corriente eléctrica, contribuyendo en el aprendizaje significativo de los estudiantes de grado 1103 del Instituto Técnico Superior de Neiva.

El diseño de mapas conceptuales y redes semánticas (Restrepo, 2013) que los estudiantes elaboraron, durante el proceso se enseñanza expositiva, convalidaron la eficacia de las estrategias usadas en el aula de clase y en el laboratorio de Física.

La evaluación del aprendizaje significativo de los estudiantes se hizo a partir de los mapas conceptuales y redes semánticas, que ellos mismos realizaron, durante el proceso se enseñanza expositiva. Los resultados de las evaluaciones infieren la eficacia de las estrategias usadas en el aula de clase y en el laboratorio de Física.

Estas estrategias didácticas también promueven el intelecto creativo, entre los estudiantes, debido a que ellas han sido usadas en la construcción de nuevo conocimiento en forma sistemática.

Las experiencias cotidianas de los estudiantes y sus conceptualizaciones previas sobre corriente eléctrica proporcionan los puntos de partida para establecer las estrategias a diseñar para procurar el aprendizaje significativo. El uso del laboratorio de Física es de vital importancia para reforzar el aprendizaje utilizando estas herramientas didácticas.

\section{Referencias Bibliográficas}

Aguilar R., Arroyo A. (2008). La metáfora científica y los libros de texto. Revista contactos. Vol. 67, p 52 - 56.

Ausubel, D. (1976). Psicología educativa. Un punto de vista cognoscitivo. Editorial Trillas. México. P 211-252.

Goldstein H. et al. (2002). Classical Mechanics. Third Edition. Addison Wesley, México.

González H. y J.P Valcárcel. Utilización del concepto de símil en la óptica geométrica. Revista Entornos No 21, p 57-62

Linares R e Izquierdo M. (2006). El rescate de la princesa encerrada en lo más alto de la torre: Un episodio para aprender sobre analogias, símiles y metáforas. Revista el Hombre y la Máquina No.27.

Marin, G. y Ortiz, R. (2014). Enseñanza del concepto de corriente eléctrica para el grado 11 basadas en analogías y metáforas. Trabajo de grado para optar el título de Licenciados en Educación Básica con énfasis en Ciencias Naturales y Educación Ambiental. Universidad Surcolombiana.

Moreira M., Greca M. (2003). Cambio conceptual: análisis crítico y propuestas a la luz de la teoría del aprendizaje significativo. Revista Ciencia e Educação, Bauru, vol. 9, n. 2, p 301-315

Novak J. y Gowin D. (1988). Mapas conceptuales para el aprendizaje significativo. En Novak J.D. et al (1998). Aprendiendo a aprender. Editorial Martínez roca. Barcelona. p. 33-57.

Tippens P. (1999). Física: Conceptos y Aplicaciones. MC Graw-Hill, Quinta Edición. México.

Pérez P. (2013), El rincón de la ciencia; revista electrónica de divulgación del I.E.S. Victoria Kent; actualizada en marzo de 2013; http://centros5.pntic.mec.es/ies.victoria.kent/RinconC/pregunta/p-19/p-19.htm

Restrepo D. (2013) Mapa conceptual física electrónica. Rescatado en octubre 09 de 2013 , en: http://cmapspublic2.ihmc.us/rid=1GGY4VW4W-164NLQYFR0/MAPA\%20CONCEPTUAL\%20FISICA\%20ELECTRONICA.cmap

Russell R. (2009). Circuitos eléctricos: analogía de agua en tubería. Rescatado en diciembre 31 de 2013 en:

http://www.windows2universe.org/physical_science/physics/el ectricity/circuit_analogy_water_pipes.htmlHYPERLINK"http://w ww.windows2universe.org/physical_science/physics/electricity/ circuit_analogy_water_pipes.html\%26lang=sp"\&HYPERLINK" http://www.windows2universe.org/physical_science/physics/el ectricity/circuit_analogy_water_pipes.html\%26lang=sp"lang=sp.

Young H. y Freedman R. (2009), Física Universitaria con Física Moderna, Editorial Pearson Educación, México; Volumen 2, decimosegunda edición.

Zamorano R., Gibbs H. y Viau J. (2006), Modelado analógico en la enseñanza de circuitos de corriente continua. Journal of Science Education, 7 (1), 30-3 